\title{
Nanoparticle-Regulated Phase Behavior of Ordered Block Copolymers
}

\author{
Michelle K. Gaines, ${ }^{\dagger}$ Steven D. Smith, ${ }^{\ddagger}$ Jon Samseth, ${ }^{\#}$ Michael R. Bockstaller ${ }^{\sharp}$ \\ Russell B. Thompson, ${ }^{\%}$ Kim Ø. Rasmussen ${ }^{\mathbf{g *}}$ and Richard J. Spontak ${ }^{\dagger *^{*}}$ \\ Departments of ${ }^{\dagger}$ Materials Science \& Engineering and ${ }^{\S}$ Chemical \& Biomolecular Engineering \\ North Carolina State University, Raleigh, NC 27695 \\ ${ }^{\ddagger}$ Miami Valley Innovation Center, The Procter \& Gamble Company, Cincinnati, OH 45061 \\ "Department of Process Technology, SINTEF Materials \& Chemistry, N-7465 Trondheim \\ and Akershus University College, N-2001 Lillestrøm, Norway \\ ${ }^{\ddagger}$ Department of Materials Science \& Engineering, Carnegie Mellon University, Pittsburgh, PA 15213 \\ ${ }^{\%}$ Department of Physics \& Astronomy, University of Waterloo, Ontario N2L 3G1, Canada \\ ${ }^{I}$ Theoretical Division, Los Alamos National Laboratory, Los Alamos, NM 87545
}

(submitted to Physical Review Letters)

This document is the accepted manuscript version of a published article. Published by The Royal Society of Chemistry in the journal "Soft Matter" issue 8, DOI: 10.1039/b805540h

\begin{abstract}
Although block copolymer motifs have received considerable attention as supramolecular templates for inorganic nanoparticles, experimental observations of a nanostructured diblock copolymer containing inorganic nanoparticles-supported by theoretical trends predicted from a hybrid self-consistent field/density functional theory-confirm that nanoparticle size and selectivity can likewise stabilize the copolymer nanostructure by increasing its order-disorder transition temperature.
\end{abstract}

\footnotetext{
* To whom correspondence should be addressed.
} 
Polymer nanocomposites remain at the forefront of advanced materials research due to the wide range of properties that can be realized by dispersing inorganic nanoparticles in a polymeric matrix at relatively low concentrations. ${ }^{1}$ In addition to improving bulk thermo-mechanical properties, ${ }^{2-5}$ nanocomposites can be used in drug encapsulation, ${ }^{6}$ optics ${ }^{7}$ and microelectronics, ${ }^{8}$ as well as in the nanoscale design of $\mathrm{Li}_{\text {batteries }}^{9}$ and gas-separation membranes. ${ }^{10,11}$ The spatial distribution of nanoparticles within the polymer constitutes a critically important consideration in nanotechnologies involving, for instance, photonic crystals, ${ }^{12}$ and efforts to meet this challenge have relied on microphase-ordered block copolymers as mesoscale templates. ${ }^{3,13-16}$ Diblock copolymer molecules consist of two long, contiguous sequences that, if sufficiently incompatible, spontaneously self-assemble to produce ordered nanostructures, viz., spheres on a bodycentered (or face-centered ${ }^{17}$ ) cubic lattice, cylinders on a hexagonal lattice, bicontinuous channels and alternating lamellae. ${ }^{18}$ Because of their ability to self-organize, copolymers with specific morphologies, dimensions or phase behavior (expressed in terms of the order-disorder transition, ODT) continue to attract attention in emerging nanotechnologies. ${ }^{19-21}$ Such systems can be tailored by either $(i)$ synthesizing molecules differing in composition or repeat unit attributes, ${ }^{22}$ or (ii) incorporating a miscible organic species (e.g., a selective solvent, parent homopolymer or second copolymer) that alters microdomain swelling and/or interfacial chain packing. ${ }^{23}$

Previous studies of spherical nanoparticles templated by microphase-ordered block copolymers have established that the spatial distribution of the nanoparticles is sensitive to factors such as concentration, selectivity and size. ${ }^{14,16,24}$ Related results that are central to the objective of the present study are summarized in Fig. 1 by segmental distributions generated from a hybrid self-consistent field (SCF) and density functional (DF) theory for an AB diblock copolymer (exhibiting the lamellar morphology) containing spherical nanoparticles. If the 
nanoparticles are sufficiently small in radius (R) relative to the size of the copolymer morphology, which is dictated by the gyration radius of the copolymer molecule $\left(\mathrm{R}_{\mathrm{g}}\right)$, they tend to distribute uniformly throughout the copolymer nanostructure and effectively behave as diluents irrespective of block selectivity ( $c f$. Figs. 1a-b and the corresponding enlargements). When the magnitude of $R / R_{g}$ is increased (Figs. 1c-d), however, block selectivity can be used to drive the nanoparticles either to the interface (neutral) or the lamellar midplane (selective), insofar as macrophase separation does not occur. ${ }^{25,26}$

While we shall return to the density distributions displayed in Fig. 1 when we discuss the physical mechanism by which nanoparticles affect copolymer phase behavior, it is important to recognize at this juncture that the addition of selective nanoparticles to a block copolymer can likewise alter how block copolymer molecules self-organize. ${ }^{26-28}$ That is, by acting as impenetrable obstacles that affect system energy and translational entropy, nanoparticles can alter chain packing ${ }^{9}$ and direct copolymer assembly, thereby altering the stability of various copolymer morphologies. This observation qualitatively agrees with earlier $\mathrm{SCF}^{29}$ studies wherein the copolymer ODT temperature $\left(\mathrm{T}_{\mathrm{ODT}}\right)$ is predicted to decrease with increasing nanoparticle concentration. Discontinuous molecular dynamics simulations ${ }^{30}$ suggest, however, that the dependence of $\mathrm{T}_{\mathrm{ODT}}$ on nanoparticle size and selectivity may be more complicated. The objectives of this study are to $(i)$ probe the phase behavior of block copolymer nanocomposites containing nanoparticles varying in concentration and selectivity and (ii) explore the phase behavior of such systems predicted by a hybrid SCF/DF theory. ${ }^{13}$

A poly(styrene- $b$-methyl methacrylate) (SM) diblock copolymer was synthesized via sequential living anionic polymerization of the $\mathrm{S}$ block in cyclohexane at $60^{\circ} \mathrm{C}$, followed by the M block in tetrahydrofuran at $-78^{\circ} \mathrm{C}$, with sec-butyllithium as the initiator. According to ${ }^{1} \mathrm{H}$ 
NMR and GPC analyses, the block masses were $13 \mathrm{kDa}$ each, and the polydispersity index was 1.05. Three grades of functionalized fumed silica (FS), obtained in powder form from Degussa Corp. (Parsippany, NJ), represented clustered nanoparticles ${ }^{9}$ varying in surface selectivity: hydroxyl-terminated $(\mathrm{OH})$, methacrylate-terminated $(\mathrm{MA})$ and octyl-terminated $(\mathrm{C} 8)$. In all cases, the primary particle size was $\sim 12 \mathrm{~nm}$. Native colloidal silica (CS-OH) particles with an average diameter of $10-15 \mathrm{~nm}$ were provided as a suspension (20\% solids) in dimethylacetamide by Nissan Chemicals (Houston, TX). Oligostyrene-coated colloidal silica (CS-OS) particles with an average core diameter of $\sim 20 \mathrm{~nm}$ were prepared by grafting oligostyrene (10 repeat units) onto colloidal silica by atom-transfer radical polymerization (ATRP). ${ }^{31}$ Specimens for rheometry were fabricated by first ultrasonicating the nanoparticles for $30 \mathrm{~min}$ in toluene, followed by copolymer dissolution and then air- and vacuum-drying, all performed at ambient temperature. No copolymer degradation due to ultrasonication was detected according to GPC analysis of the resultant films. Dynamic rheology was performed on an ARES strain-controlled rheometer operated at $1 \mathrm{rad} / \mathrm{s}$ and $2 \%$ strain amplitude to ensure linear viscoelasticity. Discs measuring 8 $\mathrm{mm}$ in diameter and $1 \mathrm{~mm}$ thick were melt-pressed at $150^{\circ} \mathrm{C}$ from dried films and heated between parallel plates to $220^{\circ} \mathrm{C}$ under nitrogen. For each material, $\mathrm{T}_{\mathrm{ODT}}$ was established from the inflection of a clearly discernible reduction in the dynamic elastic shear modulus $\left(G^{\prime}\right)$ during isochronal temperature sweeps performed at a constant cooling rate of $1^{\circ} \mathrm{C} / \mathrm{min}$.

According to dynamic rheological measurements, $\mathrm{T}_{\mathrm{ODT}}$ for the neat $\mathrm{SM}$ copolymer $\left(w_{\mathrm{p}}=0\right)$ is $186 \pm 1^{\circ} \mathrm{C}$. This temperature range is displayed as the shaded regions in Fig. 2, which also shows $\mathrm{T}_{\mathrm{ODT}}$ for the copolymer with both clustered (FS) and discrete (CS) nanoparticles. Measured values of $\mathrm{T}_{\mathrm{ODT}}$ remain relatively constant within experimental uncertainty up to $w_{\mathrm{p}}=0.01$ for all the FS-based nanoparticle aggregates, of which only the FS-C8 is shown in Fig. 2 for the sake of 
clarity. At higher nanoparticle concentrations (up to $w_{\mathrm{p}}=0.1$ ), $\mathrm{T}_{\mathrm{ODT}}$ generally decreases, with the FS-C8 series, wherein the nanoparticles are effectively neutral relative to the $\mathrm{S}$ and $\mathrm{M}$ repeat units of the copolymer, exhibiting the most pronounced reduction. Two important facts must be recognized regarding these data. The first is that, while their primary particle size is $\sim 12 \mathrm{~nm}$, FSbased nanoparticles exist for the most part (although not exclusively) as aggregate clusters that can measure up to hundreds of nanometers in size $^{9}$. At the concentrations of interest here, individual nanoparticles and small clusters are observe. In addition, the size ratio $\mathrm{R} / \mathrm{R}_{\mathrm{g}}$ must be considered. The value of $\mathrm{R}_{\mathrm{g}}$ discerned from $\sqrt{\left(\ell_{\mathrm{S}}^{2} \mathrm{~N}_{\mathrm{S}}+\ell_{\mathrm{M}}^{2} \mathrm{~N}_{\mathrm{M}}\right) / 6}$, where $\ell_{\mathrm{S}}$ and $\ell_{\mathrm{M}}$ denote the statistical segment lengths of $\mathrm{S}$ and $\mathrm{M}\left(0.71\right.$ and $0.68 \mathrm{~nm}$, respectively $\left.{ }^{32}\right)$ and $\mathrm{N}_{\mathrm{i}}(i=\mathrm{S}$ or $\mathrm{M})$ is the degree of polymerization of block $i$, is about $4.6 \mathrm{~nm}$, which translates into $\mathrm{R} / \mathrm{R}_{\mathrm{g}} \approx 1.3$ relative to the primary FS nanoparticle size.

Although these cluster and size considerations complicate interpretation, the results displayed in Fig. 2 suggest one of two scenarios at play. In the first, the FS-based nanoparticles do not induce a measurable reduction in $\mathrm{T}_{\mathrm{ODT}}$ at low $w_{\mathrm{p}}$, with selective nanoparticles (MA- and $\mathrm{OH}$ terminated) promoting a less pronounced decrease in $\mathrm{T}_{\mathrm{ODT}}$ than neutral nanoparticles at high $w_{\mathrm{p}}$. The second scenario is that the fused nanoparticle clusters aggregate ${ }^{9}$ or possibly macrophaseseparate from the copolymer, thereby leaving the copolymer matrix and its phase behavior (including $\mathrm{T}_{\mathrm{ODT}}$ ) largely unaffected at low $w_{\mathrm{p}}$. At high $w_{\mathrm{p}}$, however, entropically-unfavorable confinement effects ${ }^{33}$ adversely influence the stability of ordered copolymer molecules and lower $\mathrm{T}_{\mathrm{ODT}}$ to marginally different extents based on selectivity. The complication of fused FS clustering is altogether eliminated in the case of discrete (CS) nanoparticles, for which $T_{\mathrm{ODT}}$ is included as a function of $w_{\mathrm{p}}$ in Fig. 2. As with the FS-based nanoparticles, the CS-OH nanoparticles eventually induce a reduction in $\mathrm{T}_{\mathrm{ODT}}$ with increasing $w_{\mathrm{p}}$. Addition of the CS-OS nanoparticles, however, 
promotes a slight, but measurable, increase in $\mathrm{T}_{\mathrm{ODT}}$ up to $w_{\mathrm{p}}=0.01$, followed by a decrease thereafter. While aggregation of $\mathrm{CS}-\mathrm{OH}$ nanoparticles may again explain the reduction in $\mathrm{T}_{\mathrm{ODT}}$ apparent in Fig. 2b, the maximum in $\mathrm{T}_{\mathrm{ODT}}$ indicates that CS-OS nanoparticles can, under favorable experimental conditions, improve microphase stability. The nanoparticle characteristics capable of inducing this possibility are examined below within the framework of a hybrid SCF/DF theory ${ }^{13}$. It must be recognized, however, that the following analysis is intended to provide general physical trends and, while motivated by experimental observation, is not to be quantitatively compared to the data provided in Fig. 2.

A symmetric $A B$ diblock copolymer with $\mathrm{N}_{A}=\mathrm{N}_{B}$ is chosen to complement the experimental systems investigated here. Simulation of a copolymer/nanoparticle system commences with a random arrangement of potential fields for a copolymer of sufficiently high copolymer incompatibility $\chi_{\mathrm{AB}} \mathrm{N}$ (where $\chi_{\mathrm{AB}}$ is the Flory-Huggins interaction parameter between $\mathrm{A}$ and $\mathrm{B}$ repeat units, and $\mathrm{N}=\mathrm{N}_{\mathrm{A}}+\mathrm{N}_{\mathrm{B}}$ ) to ensure microphase-ordering into a lamellar morphology. The system is then modified with nanoparticles at a volume fraction $\phi_{\mathrm{p}}$. Incompatibility between the copolymer repeat units and nanoparticles is prescribed by assigned values of $\chi_{\mathrm{Ap}} \mathrm{N}$ and $\chi_{\mathrm{Bp}} \mathrm{N}$. For a non-selective (neutral) nanoparticle, $\chi_{\mathrm{Ap}} \mathrm{N}=\chi_{\mathrm{Bp}} \mathrm{N}$. If $\chi_{\mathrm{Ap}} \mathrm{N}<\chi_{\mathrm{Bp}} \mathrm{N}$, then the nanoparticle is considered A-selective. Upon iteration of coupled diffusion and field equations, ${ }^{34}$ the system relaxes into a nanostructure without prior knowledge of morphological symmetry. Once the copolymer nanostructure at its initially high $\chi_{\mathrm{AB}} \mathrm{N}$ is known, the value of $\chi_{\mathrm{AB}}$ is systematically reduced until a periodic nanostructure no longer develops. At this set of conditions, $\chi_{A B} N=$ $\left(\chi_{\mathrm{AB}} \mathrm{N}\right)_{\mathrm{ODT}}$. Determination of the ODT in this fashion is somewhat non-standard. Usually, the free energy of the disordered state at a given $\chi_{\mathrm{AB}} \mathrm{N}$ and $\phi_{\mathrm{p}}$ would be compared to the free energy of the ordered morphology to determine the precise location of the ODT. However, the hybrid 
SCF/DF theory employed here is not capable of rigorously resolving the ODT since the excluded volume of the particles with respect to the polymer matrix becomes increasingly inaccurate as the ODT is approached. ${ }^{35}$ Since it is not worthwhile to perform laborious free energy calculations for this reason, we have elected instead to employ the simpler procedure described above as it does yield the key variations that elucidate the effect of nanoparticles on block copolymer phase behavior.

The dependence of $\left(\chi_{A B} N\right)_{O D T}$ on $\phi_{p}$ for an $A B$ diblock copolymer modified with nanoparticles $\left(R / R_{g}=0.40\right)$ at varying levels of nanoparticle selectivity $\left(\chi_{A p} N\right)$ is presented in Fig. 3 and reveals several important features. The first is that $\left(\chi_{A B} N\right)_{\mathrm{ODT}}$ increases to a limit as the nanoparticles become less selective and $\chi_{\mathrm{Ap}} \mathrm{N}$ increases ( $c f$. the inset in Fig. 3). The second is that, for highly selective nanoparticles with $\chi_{\mathrm{AP}} \mathrm{N} \rightarrow 0,\left(\chi_{\mathrm{AB}} \mathrm{N}\right)_{\mathrm{ODT}}$ decreases with increasing $\phi_{\mathrm{p}}$ to a selectivity-dependent minimum and then increases thereafter. This minimum, which corresponds to a maximum in $\mathrm{T}_{\mathrm{ODT}}(c f$. Fig. $2 \mathrm{~b})$ and shifts to lower $\phi_{\mathrm{p}}$ with increasing $\chi_{\mathrm{Ap}} \mathrm{N}$, signifies the condition at which the energy responsible for a reduction in $\left(\chi_{A B} N\right)_{\mathrm{ODT}}$ is matched by the translational entropy that favors microphase disorder. These results most importantly establish that, if the nanoparticles are sufficiently selective, they can induce an increase in $\mathrm{T}_{\mathrm{ODT}}$, which is consistent with our experimental findings. Complementary predictions for $\left(\chi_{A B} N\right)_{O D T}$ as a function of $\phi_{\mathrm{p}}$ for A-selective nanoparticles $\left(\chi_{\mathrm{Ap}} \mathrm{N}=0\right)$ varying in size $\left(\mathrm{R} / \mathrm{R}_{\mathrm{g}}\right)$ are included in Fig. 4 , and confirm that a reduction in $\left(\chi_{\mathrm{AB}} \mathrm{N}\right)_{\mathrm{ODT}}$ is only achieved in the limit of relatively large nanoparticles (conditions corresponding to copolymer/nanoparticle macrophase separation are not considered here). In this limit, the nanoparticles tend to locate along the lamellar midplane (illustrated in Fig. 4) and stabilize the microdomains. As R/R $\mathrm{R}_{\mathrm{g}}$ decreases, $\left(\chi_{\mathrm{AB}} \mathrm{N}\right)_{\mathrm{ODT}}$ increases $(c f$. 
the inset in Fig. 4) to different extents depending on $\phi_{\mathrm{p}}$. Minima in $\left(\chi_{\mathrm{AB}} N\right)_{\mathrm{ODT}}\left(\phi_{\mathrm{p}}\right)$ are again predicted, indicating that the selectivity-induced trade-off between energetic and entropic driving forces evident in Fig. 3 is also manifested by changing the size of highly selective nanoparticles.

At a fixed $\phi_{\mathrm{p}}$, numerous small nanoparticles possess greater translational entropy than fewer large nanoparticles. As a result, the smaller nanoparticles spread to maximize their entropy. This gives rise to the segmental distributions shown in Figs. 1a and 1b, wherein the nanoparticles are almost uniformly distributed throughout the matrix. Opposing this spreading tendency is the energetic tendency for neutral nanoparticles to localize along the interface ${ }^{16}$ to relieve interfacial tension (Fig. 1a). By doing so, neutral nanoparticles screen out A/B contacts, thereby diluting the segregation and promoting an increase in $\left(\chi_{\mathrm{AB}} \mathrm{N}\right)_{\mathrm{ODT}}\left(\right.$ decrease in $\left.\mathrm{T}_{\mathrm{ODT}}\right)$ with increasing $\phi_{\mathrm{p}}$. When nanoparticles are sufficiently large, however, they possess lower translational entropy and less spreading tendency, in which case energetic considerations dominate. As seen in Fig. 1c, large neutral nanoparticles aggregate at interfaces ${ }^{14,16}$ to reduce interfacial tension by decreasing $\mathrm{A} / \mathrm{B}$ contacts and the driving force for copolymer demixing so that $\mathrm{T}_{\mathrm{ODT}}$ decreases. If small nanoparticles are selective (Fig. 1b), their translational entropy continues to dominate, and the result is that $\mathrm{T}_{\mathrm{ODT}}$ again decreases with increasing $\phi_{\mathrm{p}}$. Energy effects only prevail if the selective nanoparticles are sufficiently large and limited in translational entropy. In this limit (Fig. 1d), they localize within microdomain $\operatorname{cores}^{16}(c f$. the illustration in Fig. 4) rather than at interfaces and act as "seeds," not diluents, for the copolymer by promoting demixing and an increase in $\mathrm{T}_{\text {ODT }}$.

Contrary to the widely accepted influence of nanoparticles on ordered block copolymers, nanoparticles of appropriate size and selectivity can effectively serve as heterogeneous nucleation sites to improve the formation and stability of copolymer nanostructures that would ordinarily rely on homogeneous nucleation ${ }^{36}$ during microphase-ordering. This attribute, which 
distinguished such nanoparticles from small nanoparticles or simple low molar mass molecules that interact only by van der Waals forces, is experimentally observed and theoretically predicted. In this spirit, we note that independent studies have shown that the stability of block copolymer nanostructures can likewise be enhanced through the incorporation of selective $\mathrm{e}^{37}$ or functiona $\mathrm{l}^{38}$ homopolymers that remain mixed within (and do not macrophase-separate from) the copolymer nanostructure. Addition of selective nanoparticles to ordered block copolymers may therefore not only yield novel, spatially-modulated hybrid materials via nanoparticle assembly ${ }^{39}$ for a wide variety of growing nanotechnologies, but also provide an alternative physical means by which to promote polymeric nanostructure development.

This work was supported by the Research Council of Norway under the NANOMAT Program. Research at LANL is performed under the auspices of the National Nuclear Security Administration of the U. S. Department of Energy at Los Alamos National Laboratory under Contract No. DE-AC52-06NA25396. R. B. T. thanks the NSERC of Canada for financial support, and M. K. G. expresses her gratitude for a GEM Fellowship and a NOBCChE Procter and Gamble Fellowship. 


\section{REFERENCES}

1. R. Krishnamoorti and R. A. Vaia (eds.), Polymer Nanocomposites: Synthesis, Characterization and Modeling, American Chemical Society, Washington, D. C., 2001.

2. P. Podsiadlo et al., Science 318, 80 (2007).

3. M. R. Bockstaller, R. A. Mickiewicz and E. L. Thomas, Adv. Mater. 17, 1331 (2005).

4. A. C. Balazs, Nat. Mater. 6, 94 (2007).

5. S. C. Warren, F. J. Disalvo and U. Weisner, Nat. Mater. 6, 156 (2007).

6. R. Savić, L. Luo, A. Eisenberg and D. Maysinger, Science 300, 615 (2003).

7. L. L. Beecroft and C. K. Ober, Chem. Mater. 9, 1302 (1997).

8. D. Y. Godovsky, Adv. Polym. Sci. 153, 165 (2000).

9. F. Croce, G. B. Appetecchi, L. Persi and B. Scrosati, Nature 394, 456 (1998).

10. T. C. Merkel et al., Science 296, 519 (2002).

11. R. J. Hill, Phys. Rev. Lett. 96, 216001 (2006).

12. M. J. Fasolka et al., Phys. Rev. Lett. 90, 016107 (2003).

13. R. B. Thompson, V. V. Ginzburg, M. W. Matsen and A. C. Balazs, Science 292, 2469 (2001).

14. M. R. Bockstaller, Y. Lapetnikov, S. Margel and E. L. Thomas, J. Am. Chem. Soc. 125, $5276(2003)$.

15. A. C. Balazs, T. Emrick and T. P. Russell, Science 314, 1107 (2006).

16. R. J. Spontak et al., Nano Lett. 6, 2115 (2006).

17. T. P. Lodge, J. Bang, M. J. Park and K. Char, Phys. Rev. Lett. 92, 145501 (2004). 
18. I. W. Hamley, The Physics of Block Copolymers, Oxford University Press, New York, 1998.

19. C. Park, J. Yoon and E. L. Thomas, Polymer 44, 6725 (2003).

20. I. W. Hamley, Angew. Chem. Int. Ed. 42, 1692 (2003).

21. M. Lazzari, G. Liu and S. Lecommandoux (eds.), Block Copolymers in Nanoscience, WileyVCH, Weinheim, 2006.

22. A. K. Khandpur et al., Macromolecules 28, 8796 (1995).

23. R. J. Spontak and N. P. Patel, in Developments in Block Copolymer Science and Technology (I. W. Hamley, ed.), Wiley, New York, 2004, pp. 159-212.

24. V. Pryamitsym and V. Ganesan, Macromolecules 39, 8499 (2006).

25. S. W. Sides, B. J. Kim, E. J. Kramer and G. H. Fredrickson, Phys. Rev. Lett. 96, 250601 (2006).

26. C.-T. Lo, B. Lee, V. G. Pol, N. L. Dietz Rago, S. Seifert, R. E. Winans and P. Thiyagarajan, Macromolecules, 2007, 40, 8302.

27. B. J. Kim et al., Adv. Mater. 17, 2618 (2005).

28. B. J. Kim, G. H. Fredrickson, C. J. Hawker and E. J. Kramer, Langmuir 23, 7804 (2007).

29. A. I. Chervanyov and A. C. Balazs, J. Chem. Phys. 119, 3529 (2003).

30. A. J. Schultz, C. K. Hall and J. Genzer, Macromolecules 38, 3007 (2005).

31. ATRP

32. F. Rodriguez, C. Cohen, C. K. Ober and L. A. Archer, Principles of Polymer Systems, $5^{\text {th }}$ Ed., Taylor \& Francis, New York, 2003, p. 264.

33. P. Rittigstein, R. D. Priestley, L. J. Broadbelt and J. M. Torkelson, Nat. Mater. 6, 278 (2007).

34. K. O. Rasmussen and G. Kalosakas, J. Polym. Sci. B: Polym. Phys. 40, 1777 (2002). 
35. M. W. Matsen and R. B. Thompson, Macromolecules (submitted).

36. W. G. Kim, M. Y. Chang, B. A. Garetz, M. C. Newstein, N. P. Balsara, J. H. Lee, H. Hahn and S. S. Patel, J. Chem. Phys., 2001, 114, 10196.

37. M. W. Matsen, Macromolecules, 2003, 36, 9647.

38 V. R. Tirumala, A. Romang, S. Agarwal, E. K. Lin and J. J. Watkins, Adv. Mater., 2008, DOI: 10.1002/adma.200701577.

39 A. J. Rahedi, J. F. Douglas and F. W. Starr, J. Chem. Phys., 2008, 128, 024902. 


\section{List of Figure Captions}

Figure 1. Segmental density profiles of the A block (solid line), B block (dotted line) and nanoparticle ( $\mathrm{p}$, bold solid line) as a function of distance $(x)$ perpendicular to the lamellar microdomains in an ordered block copolymer and normalized with respect to the microdomain period $(D)$. These results are generated by a hybrid SCF/DF theory for AB diblock copolymers $\left(\chi_{\mathrm{AB}} \mathrm{N}=25, \mathrm{~N}_{\mathrm{A}}=\mathrm{N}_{\mathrm{B}}\right)$ modified with $(\mathrm{a}, \mathrm{b})$ small $\left(\mathrm{R} / \mathrm{R}_{\mathrm{g}}=0.05\right)$ and $(\mathrm{c}, \mathrm{d})$ large $\left(\mathrm{R} / \mathrm{R}_{\mathrm{g}}=0.50\right)$ nanoparticles varying in selectivity: $(a, c)$ neutral $\left(\chi_{A p} N=\chi_{B p} N\right)$ and $(b, d)$ A-selective $\left(\chi_{A p} N=0\right)$. The enlargements below (a) and (b) show the corresponding nanoparticle density profiles.

Figure 2. Dependence of the order-disorder transition temperature $\left(\mathrm{T}_{\mathrm{ODT}}\right)$ measured by isochronal dynamic rheology on nanoparticle concentration $\left(\phi_{p}\right)$ for (a) clustered nanoparticles and (b) discrete nanoparticles varying in surface chemistry, which governs block selectivity (labeled). The solid lines serve as guides for the eye, and the shaded region corresponds to the range over which $\mathrm{T}_{\mathrm{ODT}}$ of the neat copolymer has been measured.

Figure 3. Predicted dependence of $\left(\chi_{\mathrm{AB}} \mathrm{N}\right)_{\mathrm{ODT}}$ from SCF/DF analysis on the volume fraction of nanoparticles $\left(\phi_{\mathrm{p}}\right)$, which vary in block selectivity (values of $\chi_{\mathrm{Ap}} \mathrm{N}$ are labeled and $\chi_{\mathrm{Bp}} \mathrm{N}=\chi_{\mathrm{AB}} \mathrm{N}$ ) at $\mathrm{R} / \mathrm{R}_{\mathrm{g}}=0.40$. The dashed line identifies $\left(\chi_{\mathrm{AB}} \mathrm{N}\right)_{\mathrm{ODT}}$ for the neat copolymer, and the arrowheads denote minima in the curves. The inset shows the explicit effect of nanoparticle selectivity on copolymer phase behavior at two different concentrations $\left(\phi_{\mathrm{p}}=0.05\right.$ and 0.10$)$.

Figure 4. Predicted dependence of $\left(\chi_{\mathrm{AB}} \mathrm{N}\right)_{\mathrm{ODT}}$ from SCF/DF analysis on $\phi_{\mathrm{p}}$ for A-selective nanoparticles $\left(\chi_{\mathrm{Ap}} \mathrm{N}=0\right.$ and $\left.\chi_{\mathrm{Bp}} \mathrm{N}=\chi_{\mathrm{AB}} \mathrm{N}\right)$ varying in $\mathrm{R} / \mathrm{R}_{\mathrm{g}}($ in 0.04 intervals, labeled). The inset 
shows the explicit effect of nanoparticle size on copolymer phase behavior at two different nanoparticle concentrations $\left(\phi_{\mathrm{p}}=0.05\right.$ and 0.10$)$, and the illustration depicts the observed ${ }^{16}$ localization of nanoparticles in the large $\mathrm{R} / \mathrm{R}_{\mathrm{g}}$ limit. 
Gaines et al.
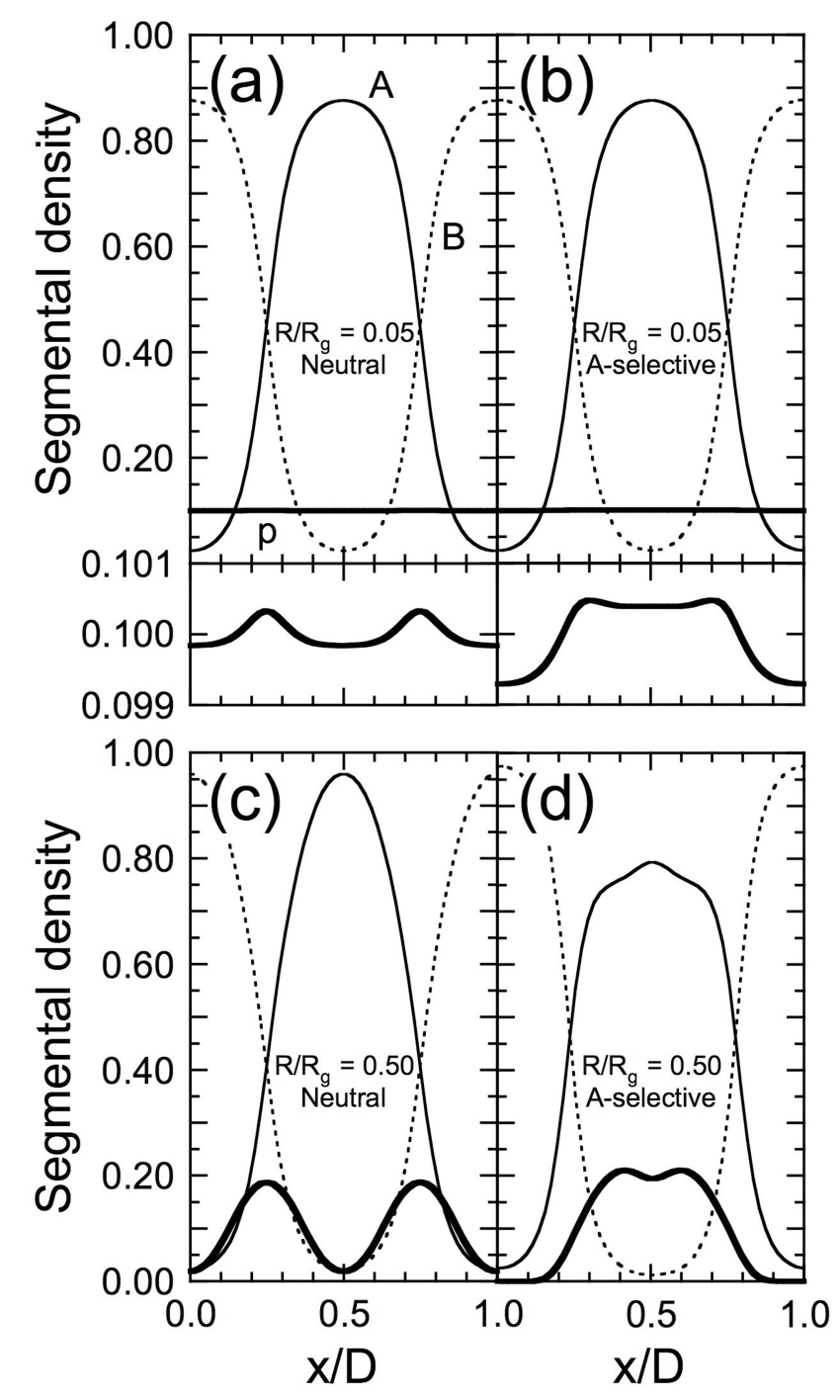

Figure 1. Gaines et al. 


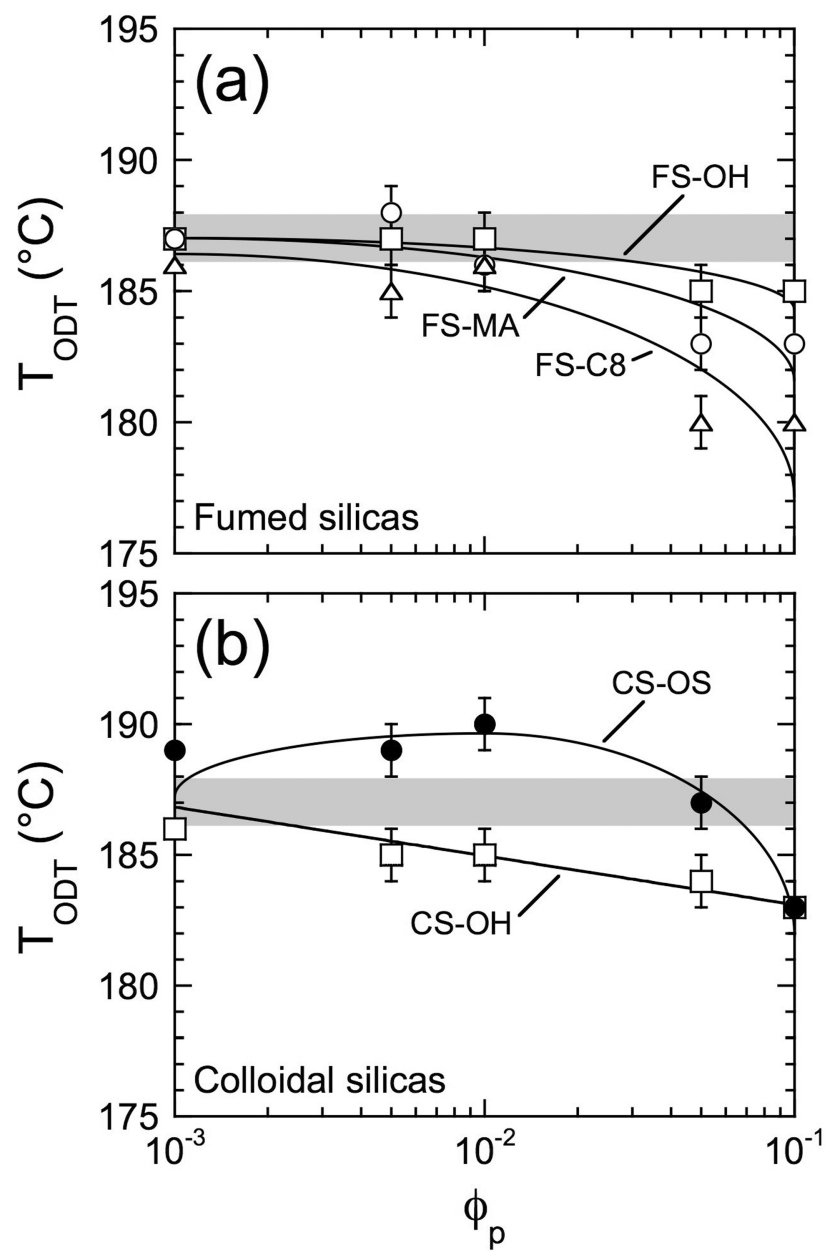

Figure 2. Gaines et al. 


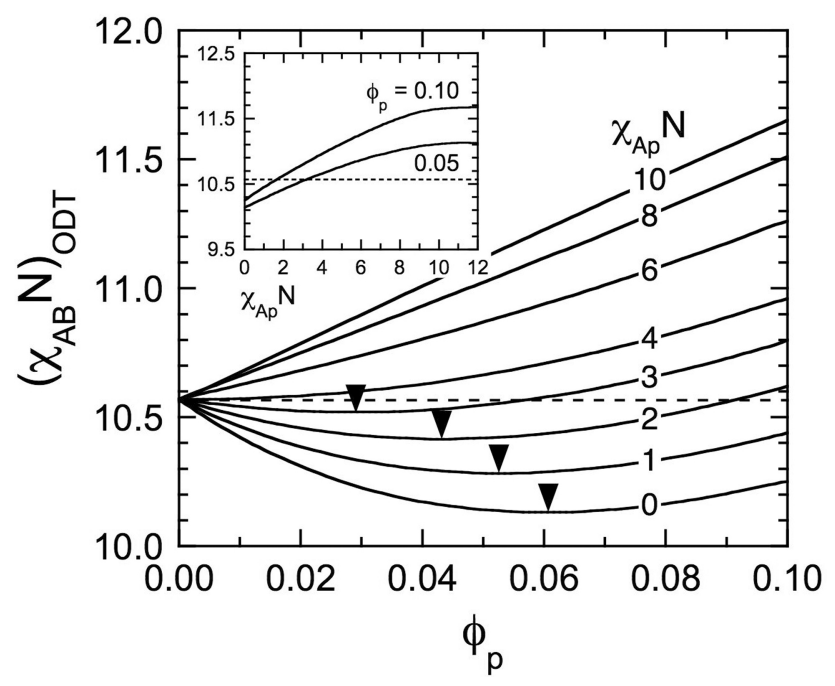

Figure 3. Gaines et al.

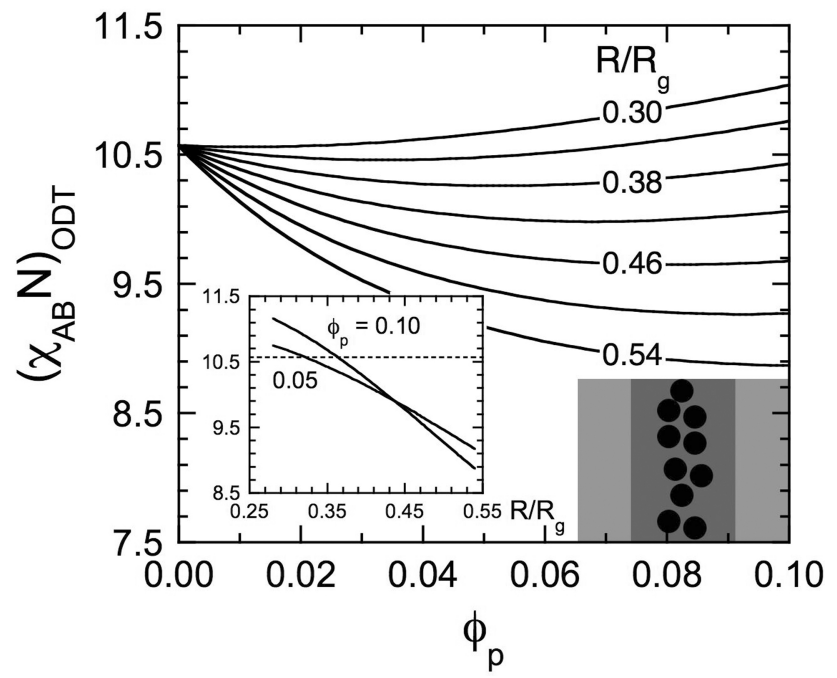

Figure 4. Gaines et al. 